\title{
Study of Higgs Phenomenology in the Next-to-Minimal Supersymmetric Standard Model (NMSSM)
}

\author{
Partha Pratim Pal \\ BolpurSikshaniketan Ashram Vidyalaya, West Bengal, Bolpur, Birbhum, India
}

Email address:

partha4321@yahoo.com (P.P. Pal)

To cite this article:

Partha Pratim Pal. Study of Higgs Phenomenology in the Next-to-Minimal Supersymmetric Standard Model (NMSSM). International Journal of High Energy Physics. Vol. 2, No. 3, 2015, pp. 34-37. doi: 10.11648/j.ijhep.20150203.11

\begin{abstract}
The theoretical and phenomenological aspects of the Next-to-Minimal Supersymmetric Standard Model (NMSSM) is reviewed. The model includes an additional gauge singlet $S$ which generates the $\mu$ - term dynamically and the $\mu$ - problem in MSSM is solved. The different masses of seven Higgs bosons and the bounds of the mass parameters have been discussed.
\end{abstract}

Keywords: NMSSM, MSSM, Gauge Singlet S, $\mu$ - Problem, Mass Parameters

\section{Introduction}

Supersymmetry[1,2] is one of the most appealing concepts of Physicsbeyond the Standard Model(SM). The main motivation of Supersymmetry is to stabilize the hierarchy[3] between the electroweak and the GUT or Plank scale. In the Standard Model (SM), the Higgs boson mass gains radiative corrections which depend quadratically on the cut-off scale of the theory, threatening to generate a mass which is far too large to explain electroweak symmetry breaking. The supersymmetry ensures that the contribution of new supersymmetric particles exactly cancel the quadratic divergence of their Standard Model partners, removing the sensitivity of the Higgs mass to the cut off scale in a natural way. In this way Supersummetry stabilizes the Higgs mass at a phenomenologically acceptable scale (below TeV). The MSSM contains a $\mu$ - parameter in the superpotential where $\mu$ is a dimensionful parameter. It enters the Higgs potential with the soft scalar masses to determine the vacuum expectation value of the Higgs fields. This parameter has to be adjusted by hand to a value at the electroweak scale, in order to provide the correct pattern of electroweak symmetry breaking. This is a problem of the model and is called $\mu$-problem[4]. In the NMSSM $[5,6,7]$ an additional gauge singlet $\mathrm{S}$ (the complex scalar component of a chiral superfield $\hat{S}$ ) is introduced which generates the $\mu$-term dynamically, i.e. an effective $\mu$-term arises spontaneously and the adjustment by hand drops out. The effective $\mu$-parameter may then naturally be expected to be of the electroweak scale, $\mu=\lambda\langle S\rangle$.In this sense the NMSSM is a natural extension of the MSSM.

The superpotential for the NMSSM is given by

$$
W_{N M S S M}=W_{Y U K}+\lambda S H_{u} H_{d}+\frac{k}{3} S^{3}
$$

Where $W_{Y U K}$ gives the Yukawa couplings for the Standard Model fermions. Without the $\mathrm{k}$ term the superpotential would have a $U(1)^{/}$symmetry, so called Peccei-Quinn symmetry[8]. This additional symmetry would alter the phenomenology completely. The role of the $\mathrm{k}$ term is to break this $U(1)^{\prime}$ symmetry. The $\mathrm{k}$ term is introduced trilinear such that $\mathrm{k}$ is dimensionless. However, there remains a discrete $Z_{3}$ symmetry[9,10] which is moreover broken spontaneously. In principal this leads to the domain wall problem. Introducing additional, but suppressed terms, the $Z_{3}$ symmetry can be broken without changing phenomenology at the electroweak scale. It is assumed that the domain wall problem is circumvented in this way without any modifications except far beyond the electroweak scale.Thus NMSSM provides a lot of new phenomenological aspects which is absent in SM [11] and $\operatorname{MSSM}[12]$.

However, the Higgs bosons in MSSM and NMSSM are given in the table below 
Table 1. Higgs bosons in MSSM and NMSSM.

\begin{tabular}{llllll}
\hline Model & Symmetry & Superpotential & CP-even & CP-odd & Charged \\
\hline MSSM & - & $\mu H_{u} H_{d}$ & $H_{1}^{0}, H_{2}^{0}$ & $A^{0}$ & $H^{ \pm}$ \\
NMSSM & $Z_{3}$ & $\lambda S H_{u} H_{d}+\frac{k}{3} S^{3}$ & $H_{1}^{0}, H_{2}^{0}, H_{3}^{0}$ & $A_{1}^{0}, A_{2}^{0}$ & $H^{ \pm}$ \\
\hline
\end{tabular}

The paper is organized as follows: Section 2 gives the expressions of the Higgs potential in NMSSM. Tadpole conditions are discussed in section 3 . The expressions of the masses of Higgs scalars in NMSSM is given in section 4. In section 5 various parameter constraints have been discussed. The conclusions are given in section 6 .

\section{The Higgs Potential in NMSSM}

The Higgs fields of the NMSSM consists of the usual two Higgs doublets as in the MSSM together with an extra Higgs singlet,

$$
\begin{array}{r}
H_{u}=\left(\begin{array}{c}
H_{u}^{+} \\
H_{u}^{0}
\end{array}\right), H_{d}=\left(\begin{array}{l}
H_{d}^{0} \\
H_{d}^{-}
\end{array}\right), S \quad V_{D}=\frac{g^{2}+g}{8}\left(\left|H_{d}\right|^{2}-\left|H_{u}\right|^{2}\right)^{2}+\frac{1}{2} g^{2} \\
V_{\text {Soft }}=m_{H_{u}}^{2}\left|H_{u}\right|^{2}+m_{H_{d}}^{2}\left|H_{d}\right|^{2}+m_{S}^{2}|S|^{2}+\left[\lambda A_{\lambda} S H_{u} H_{d}+\frac{1}{3} k A_{k} S^{3}+\text { h.c. }\right]
\end{array}
$$
mixing with the other Higgs fields. parts,

$$
V=V_{F}+V_{D}+V_{S o f t}
$$

With,
The extra singlet is allowed to couple only to the Higgs doublets of the model, and consequently the couplings of the new fields to gauge bosons will only be manifested via their

The tree-level Higgs potential[10] is composed of three

$$
\begin{gathered}
V_{F}=|\lambda S|^{2}\left(\left|H_{u}\right|^{2}+\left|H_{d}\right|^{2}\right)+\left|\lambda H_{u} H_{d}+k S^{2}\right|^{2} \\
V_{D}=\frac{g^{2}+g^{\prime 2}}{8}\left(\left|H_{d}\right|^{2}-\left|H_{u}\right|^{2}\right)^{2}+\frac{1}{2} g^{2}\left|H_{u}^{+} H_{d}\right|^{2}
\end{gathered}
$$

where $g$ and $g /$ being the gauge couplings of $S U(2)_{L}$ and $U(1)$ interactions respectively. The Higgs potential contains seven parameters: $\lambda$ and $\mathrm{k}$ from the superpotential and $A_{k}, A_{\lambda}, m_{H_{u}}, m_{H_{d}}$ and $m_{s}$ from the soft supersymmetry breaking terms.

\section{Tadpole Conditions}

Let us expand the potential about the VEVs represented by $v_{u}, v_{d}, v_{s}$ and the phases $\theta$ and $\varphi$. The parameterization of the scalar fields is as follows:

$$
H_{d}=\left(\begin{array}{c}
\frac{1}{\sqrt{2}}\left(v_{d}+h_{d}+i a_{d}\right) \\
H_{d}^{-}
\end{array}\right), H_{u}=e^{i \theta}\left(\begin{array}{c}
H_{u}^{+} \\
\frac{1}{\sqrt{2}}\left(v_{u}+h_{u}+i a_{u}\right)
\end{array}\right), S=\frac{1}{\sqrt{2}} e^{i \varphi}\left(v_{s}+h_{s}+i a_{s}\right)
$$

Where we have taken the vacuum-expectation values $v_{u}, v_{d}, v_{s}$ to be real and non-negative. The values $v_{u}, v_{d}$ and $v_{s}$ will take the values for which the potential V in equation(3) has a global minimum. In the parameterization (7) the vacuum denoted by $<>$ precisely means

$$
\left\langle H_{d}\right\rangle=\left(\begin{array}{c}
\frac{v_{d}}{\sqrt{2}} \\
0
\end{array}\right),\left\langle H_{u}\right\rangle=e^{i \theta}\left(\begin{array}{c}
0 \\
\frac{v_{u}}{\sqrt{2}}
\end{array}\right),\langle S\rangle=e^{i \varphi} \frac{v_{S}}{\sqrt{2}}
$$

The condition for the scalar potential to have an extremum at the vacuum is that the first derivatives with respect to the Higgs fields evaluated at the vacuum vanishes

$$
\begin{aligned}
& 0=\frac{1}{v_{d}}\left\langle\frac{\partial V}{\partial h_{d}}\right\rangle=m_{H_{d}}^{2}-R_{\lambda} \frac{v_{u} v_{s}}{v_{d}}+\frac{g^{2}+g^{\prime 2}}{8}\left(v_{d}^{2}-v_{u}^{2}\right)+\frac{|\lambda|^{2}}{2}\left(v_{u}^{2}+v_{s}^{2}\right)-\frac{R}{2} \frac{v_{u} v_{s}^{2}}{v_{d}} \\
& 0=\frac{1}{v_{u}}\left\langle\frac{\partial V}{\partial h_{u}}\right\rangle=m_{H_{u}}^{2}-R_{\lambda} \frac{v_{d} v_{s}}{v_{u}}+\frac{g^{2}+g^{\prime 2}}{8}\left(v_{d}^{2}-v_{u}^{2}\right)+\frac{|\lambda|^{2}}{2}\left(v_{d}^{2}+v_{s}^{2}\right)-\frac{R}{2} \frac{v_{d} v_{s}^{2}}{v_{u}} \\
& 0=\frac{1}{v_{s}}\left\langle\frac{\partial V}{\partial h_{s}}\right\rangle=m_{s}^{2}-R_{\lambda} \frac{v_{d} v_{u}}{v_{s}}+R_{k} v_{s}+\frac{|\lambda|^{2}}{2}\left(v_{d}^{2}+v_{u}^{2}\right)+|k|^{2} v_{s}^{2}-R v_{d} v_{u}
\end{aligned}
$$

$0=\frac{1}{v_{u}}\left\langle\frac{\partial V}{\partial a_{d}}\right\rangle=\frac{1}{v_{d}}\left\langle\frac{\partial V}{\partial a_{u}}\right\rangle=I_{\lambda} v_{s}-\frac{1}{2} I v_{s}^{2}$

$0=\frac{1}{v_{s}}\left\langle\frac{\partial V}{\partial a_{s}}\right\rangle=I_{\lambda} \frac{v_{d} v_{u}}{v_{s}}+I_{k} v_{s}+I v_{d} v_{u}$

Where the introduced abbreviations are

$$
\begin{aligned}
R & =\operatorname{Re}\left[\lambda k^{*} e^{i(\theta-2 \varphi)}\right] \\
I & =I_{m}\left[\lambda k^{*} e^{i(\theta-2 \varphi)}\right]
\end{aligned}
$$




$$
\begin{aligned}
& R_{\lambda}=\frac{1}{\sqrt{2}} \operatorname{Re}\left[\lambda A_{\lambda} e^{i(\theta+\varphi)}\right] \\
& I_{\lambda}=\frac{1}{\sqrt{2}} I_{m}\left[\lambda A_{\lambda} e^{i(\theta+\varphi)}\right] \\
& R_{k}=\frac{1}{\sqrt{2}} \operatorname{Re}\left[k A_{k} e^{i 3 \varphi}\right] \\
& I_{k}=\frac{1}{\sqrt{2}} I_{m}\left[k A_{k} e^{i(\theta+\varphi)}\right]
\end{aligned}
$$

These conditions are called tadpole conditions in the sense that the conditions make the tadpole diagrams vanish if we set the Higgs fields to their VEVs. Here $\mathrm{R}$ and $\mathrm{I}$ are dimensionless parameters and $R_{\lambda}, R_{k}, I_{\lambda}$ and $I_{k}$ have the dimension of mass.

From $\mathrm{e} q^{n}$ (12) and (13), the following two conditions are obtained-

$$
\begin{gathered}
I_{\lambda}=-\frac{1}{2} I v_{s} \\
\left.I_{k}=-\frac{3}{2} I \frac{v_{d} v_{u}}{v_{s}}\right\} \\
M_{H_{2,1}}^{2}=\frac{1}{2}\left\{M_{Z}^{2}+\frac{1}{2} k v_{s}\left(4 k v_{s}+\sqrt{2} A_{k}\right) \pm \sqrt{\left[M_{Z}^{2}-\frac{1}{2} k v_{s}\left(4 k v_{s}+\sqrt{2} A_{k}\right)\right]^{2}+\cot ^{2} \beta_{s}\left[2 \lambda^{2} v_{s}^{2}-M_{A}^{2} \operatorname{Sin}^{2} 2 \beta\right]^{2}}\right\}
\end{gathered}
$$

Only one of the three imaginary parts $\mathrm{I}, I_{\lambda}, I_{k}$ is physical, when complex parameters are introduced, they must be chosen to satisfy the tadpole conditions (20).

\section{The Mass of Higgs Scalars}

In the NMSSM seven Higgs bosons are created: $3 \mathrm{CP}$ even $\left(H_{1}^{0}, H_{2}^{0}, H_{3}^{0}\right)$, two CP-odd $\left(A_{1}^{0}, A_{2}^{0}\right)$ and two charged $\left(H^{ \pm}\right)$. Their tree level masses are given below[10]

$$
\begin{gathered}
M_{H^{ \pm}}^{2}=M_{A}^{2}+M_{W}^{2}-\frac{1}{2}(\lambda v)^{2} \\
M_{A_{2}}^{2}=M_{A}^{2}\left(1+\frac{1}{4} \cot ^{2} \beta_{S} \operatorname{Sin}^{2} 2 \beta\right) \\
M_{A_{1}}^{2}=-\frac{3}{\sqrt{2}} k v_{S} A_{k} \\
M_{H_{3}}^{2}=M_{A}^{2}\left(1+\frac{1}{4} \cot ^{2} \beta_{S} \operatorname{Sin}^{2} 2 \beta\right)
\end{gathered}
$$

Where $\quad v=\sqrt{v_{u}^{2}+v_{d}^{2}}=246 \mathrm{GeV}, \tan \beta=\frac{v_{u}}{v_{d}}, \tan \beta_{s}=\frac{v_{s}}{v}$ and negative value for $A_{k}$ being preferred.

It can be noted that the heavy CP-odd Higgs boson $A_{2}$ is degenerate with the heaviest CP-even Higgs boson $\mathrm{H}_{3}$.

Moreover,

$$
M_{H_{1}}^{2}+M_{H_{2}}^{2}=M_{z}^{2}+\frac{1}{2} k v_{s}\left(4 k v_{s}+\sqrt{2} A_{k}\right)
$$

coupling $\lambda$ and $M_{A}^{2}$.

\section{Parameter Constraints}

There are seven parameters in the Higgs potential- $\lambda$ and $\mathrm{k}$ from the superpotential and $A_{\lambda}, A_{k}, m_{H_{d}}, m_{H_{u}}, m_{s}$ from the soft supersymmetry breaking terms. The field values at $v_{u}, v_{d}$ and $v_{s}$ are given by the following equations[10]

The sum of the two lightest scalars, is independent of the

$$
\begin{gathered}
m_{H_{d}}^{2}=\frac{\left(g^{2}+g^{\prime 2}\right)}{8}\left(v_{u}^{2}-v_{d}^{2}\right)-\frac{1}{2} \lambda^{2} v_{u}^{2}+\frac{1}{2}\left(\sqrt{2} A_{\lambda}+k v_{s}\right) \lambda v_{s} \frac{v_{u}}{v_{d}}-\frac{1}{2} \lambda^{2} v_{s}^{2} \\
m_{H_{u}}^{2}=\frac{\left(g^{2}+g^{\prime 2}\right)}{8}\left(v_{d}^{2}-v_{u}^{2}\right)-\frac{1}{2} \lambda^{2} v_{d}^{2}+\frac{1}{2}\left(\sqrt{2} A_{\lambda}+k v_{s}\right) \lambda v_{s} \frac{v_{d}}{v_{u}}-\frac{1}{2} \lambda^{2} v_{s}^{2} \\
m_{s}^{2}=-k^{2} v_{s}^{2}-\frac{1}{2} \lambda^{2} v^{2}+k \lambda v_{u} v_{d}+\frac{1}{\sqrt{2}} \lambda A_{\lambda} \frac{v_{u} v_{d}}{v_{s}}-\frac{1}{\sqrt{2}} k A_{k} v_{s}
\end{gathered}
$$

The renormalization group equations for $\lambda$, $\mathrm{k}$ and top Yukawa coupling $h_{t}$ are related to the gauge couplings and are given by

$$
\begin{gathered}
16 \pi^{2} \frac{d g_{i}^{2}}{d t}=b_{i} g_{i}^{2} \\
16 \pi^{2} \frac{d h_{t}^{2}}{d t}=h_{t}^{2}\left[\lambda^{2}+6 h_{t}^{2}-\frac{16}{3} g_{3}^{2}-3 g_{2}^{2}-\frac{13}{15} g_{1}^{2}\right] \\
16 \pi^{2} \frac{d \lambda^{2}}{d t}=\lambda^{2}\left[4 \lambda^{2}+2 k^{2}+3 h_{t}^{2}-3 g_{2}^{2}-\frac{3}{5} g_{1}^{2}\right] \\
16 \pi^{2} \frac{d k^{2}}{d t}=6 k^{2}\left[\lambda^{2}+k^{2}\right]
\end{gathered}
$$

Here, $\quad b_{1}=\frac{33}{5}, b_{2}=1, g_{1}=\sqrt{\frac{5}{3}} g^{\prime}, g_{2}=$ gandt $=$ $\log \frac{Q^{2}}{M_{G U T}^{2}}$ where $\mathrm{Q}$ is the renormalization scale.
Values of $\lambda$ and $\mathrm{k}$ in the perturbative regime at the GUT scale is given by $\frac{\lambda}{2 \pi} \leq 1, \frac{k}{2 \pi} \leq 1$. But $\lambda$ and $\mathrm{k}$ are reduced to small values at the electroweak scale and their combined approximate bound is given by [10]

$$
\lambda^{2}+k^{2} \leq 0.5
$$

$\lambda$ must not become too small. If $\lambda$ becomes too small then the phenomenological constraints on $\mu$ require $v_{s}$ to be large and the link with the other Higgs VEVs is lost. A small value of $\tan \beta$ is favoured and it is kep $t \leq 10 . v_{s}$ is expected to vary from $3 v$ to $15 v$.

The value of $A_{k}$ is determined from the requirement of vacuum stability. Positive and large value of $A_{k}$ destabilizes the vacuum and the mass squared of the lightest pseodoscalar becomes negative. The soft SUSY breaking term containing $A_{k}$ is a cubic coupling proportional to $S^{3}$, so the effect of varying 
$A_{k}$ is only communicated through the singlet contribution of the Higgs fields. The heavy Higgs bosons and one of the lighter scalars are very insensitive to the choice of $A_{k}$.

$A_{\lambda}$ is also constrained by vacuum stability and its natural value is approximately $\mu \tan \beta$.

\section{Conclusions}

The present paper investigates the Higgs sector of the Nextto-Minimal Supersymmetric Standard Model. In this model the $\mu$ problem of MSSM is solved by introducing a new singlet Higgs field S. The expressions of the Higgs boson masses have been introduced and it provides a nice insight into the mass hierarchies. The present paper also throws light into the tadpole condition. The MSSM limit can be regained by letting $\lambda$ and $\mathrm{k} \rightarrow 0$ while keeping $\mu, A_{\lambda}$ and $A_{k}$ fixed. The bounds of the mass parameters have been discussed. The requirement of vacuum stability provides useful bounds on the mass parameters. The lightest Higgs boson in the NMSSM is heavier than the one of the MSSM due to additional terms in the tree level Higgs potential proportional to $\lambda^{2}$; the additional contribution is $\Delta m_{h}^{2}=\frac{\lambda^{2}}{g^{2}} M_{Z}^{2} \operatorname{Sin}^{2} 2 \beta$. It is obvious that the upper bound on the mass of the lightest Higgs boson is expected to be larger than that in the MSSM.

\section{References}

[1] J. L. Gervais and B. Sakita, Nucl. Phys. B 34 (1971) 632; J. Wess and B. Zumino, Nucl. Phys. B 70 (1974) 39; D. V. Volkov and V. P. Akulov, Phys. Lett. B 46 (1973) 109.
[2] H. E. Haber and G. L. Kane, Phys. Rept. 117 (1985) 75; J. F. Gunion and H. E. Haber, Nucl. Phys. B 272 (1986) 1; G. R. Farrar and P. Fayet, Phys. Lett. B 76 (1978) 575.

[3] N. Sakai, Z. Phys. C 11(1981)153; J. Polchinski and L. Susskind, Phys. Rev. D 26 (1982) 3661.

[4] J. E. Kim and H. P. Nilles, Phys. Lett. B 138, 150 (1984).

[5] M. Bastero-Gil, C. Hugonic, S. F. King, D. P. Roy and S. Vempati, Phys. Lett. B 489, 359 (2000); A. de Gouvea, A. Friedland and H. Murayama, Phys. Rev. D 57, 5676 (1998).

[6] J. Ellis, J. F. Gunion, H. Haber, L. Roszkowski, F. Zwirner, Phys. Rev. D 39 (1989) 844.

[7] J. P. Derendinger and C. A. Savoy, Nucl. Phys. B 237 (1984) 307;J. M. Frere, D. R. Jonesand S. Raby, Nucl. Phys. B 222(1983) 11.

[8] R. D. Peccei and H. R. Quinn, Phys. Rev. Lett. 38 (1977) 1440; Phys. Rev. D 16 (1977) 1791.

[9] J. R. Ellis, J. F. Gunion, H. E. Haber, L. Roszkowski and F. Zwirner, Phys. Rev. D 39, 844 (1989); S. W. Ham, S. K. Oh, and B. R. Kim, J. Phys. G 22, 1575 (1996).

[10] D. J. Miller, R. Nevzorov and P. M. Zerwas, Nucl. Phys. B 681, 3 (2004).

[11] P. P. Pal, American Journal of Physics and Applications, 2015; 3(2):63-66. DOI: 10. 11648/j. ajpa. 20150302. 18.

[12] P. P. Pal, American Journal of Physics and Applications, 2015;3(2):57-59. DOI: 10. 11648/j. ajpa. 20150302. 16. 